\title{
BRITE-Constellation: Astrophysical Concept
}

\author{
W. Weiss ${ }^{1}$
}

${ }^{1}$ Institut für Astronomie, Türkenschanzstrasse 17, 1180 Vienna, Austria

\section{Abstract}

This proceeding paper was generated using a Power-Point presentation from the workshop.

\section{Presentation Slides}

Many technical and scientific details of BRITE-Constellation will be discussed during this workshop. Hence, I can limit myself to briefly summarize the arguments leading to the concept of BRITE-Constellation.

\section{4 "elements" of BRITE-Constellation}

1. Science case: Variability of (massive) bright stars in time scale of hours and more \& extensive additional science

2. Feasibility: Nano-sat CanX program at UTIAS, miniaturized nanoHPAC stabilisation system (based on MOST experience) the Canadian connection

3. Funding in Austria:

1. UNIBRITE by U-Vienna (2005), built by UTIAS-SFL

2. BRITE-Austria (TUG Sat-1) by ASAP3 (2005) and ASAP4 (2006) as first Austrian Satellite, built in and operated from Austria by TU-Graz

3. Canadian counterpart (tbc) BRITE-Canada 1 \& 2

4. Innovation: Operating a system of nanosats on a national level: BRITE-Constellation. proposed by If $A$

HPAC: High Precision Attitude Control (system); UTIAS: University of Toronto, Institute for Aerospace Studies; SFL: Space Flight Laboratory; ASAP: Austrian Space \& Aeronautic Program. 


\section{Why from space?}

- High photometric precision for bright stars not realistic from ground

- Complementary to MOST \& COROT

- FOV of $25 \times 25^{\circ} \rightarrow$ average 4 stars $+3.5^{\mathrm{m}}$ in Milky Way 6-16 stars (1/3 sky with >2 stars)

- Potentially strong public interest

- Amateurs \& schools (ground support). and parallel spectroscopy is realistic !!!

\section{To be kept in mind during this workshop}

- Low budget space project (Mission requirements)

- equivalent of typically $1 / 10 \mathrm{VLT}$ backinstruments or 1/400 ESA Medium Size Mission

- Limits in science potential (importance of optimization)

- Yet not fully decided

- Launch date

- Orbit

- 1 or 2 pairs (Canada / Spain / Russia) 
The next slide illustrates three extreme LEOs (Low Earth Orbits) with distinct advantages and disadvantages for spacecraft operation and observing programs. From the polar orbits with continuous viewing zones (CVZs) along the celestial equator we show a dusk-dawn orbit (realized for MOST) with the advantage of a rather constant thermal load and stray light situation. This

\section{Dusk-Dawn, Midnight-Noon, Aequatorial}

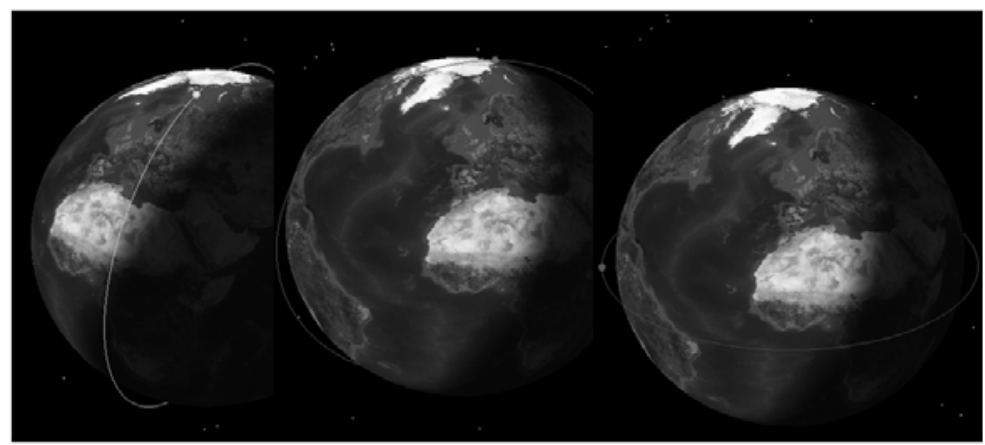

will be certainly not the case for a midnight-noon orbit. However, as can be seen from the next figure, stray light variations for a dusk-dawn orbit still can be strong. Good baffling will therefore be a requirement for BRITE, but sophisticated data reduction can reduce the stray light component significantly (next-but-one figure).

The CoRoT orbit is sun-synchronous and changing during about 3 months from dusk-dawn to midnight-noon.

The equatorial orbit provides CVZs at the celestial poles, but for a low orbit the satellite would not be visible from Vienna preventing telecommunication. The MOST orbit is a dusk-dawn orbit. 


\section{MOST stray light}

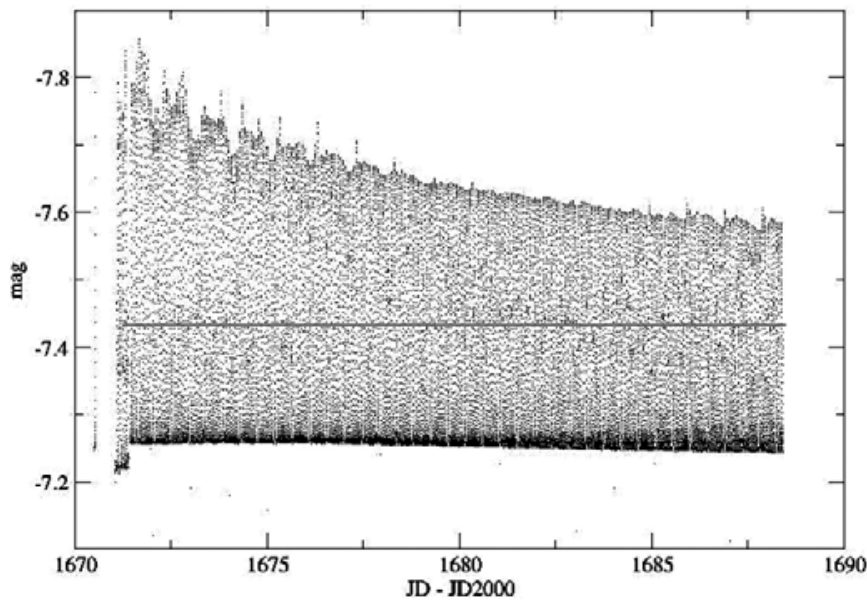

\section{MOST stray light corrected}

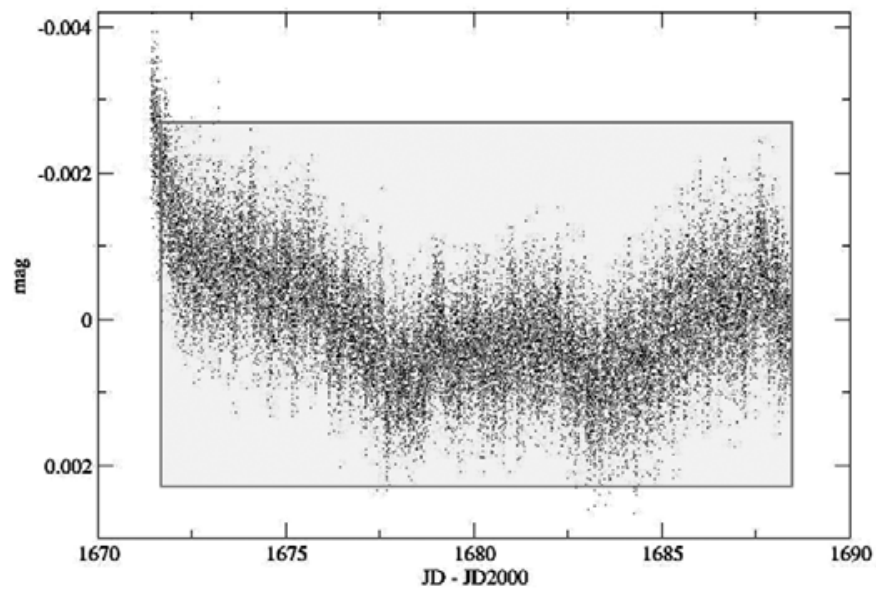






This figure illustrates the advantage of uninterrupted observations (from space). It shows Fourier spectra of simulated data containing white noise with an amplitude of 1 and three sinusoidal signals with same amplitude of 1 (vertical arrows). The inserted figure in the upper right corner indicates the Spectral Window of the simulated data set.

For a single BRITE satellite and one data point per orbit (of typically 90 min orbit period) integrated during 15 minutes one would not be able to detect the input signal due to the high noise level. The highest frequency signal could not be detected as it is beyond the Nyquist frequency.

The noise level in the Fourier domain can be drastically reduced, if the observations can be extended from only 6 days to 6 months (lower left panel). An increased sensitivity by a factor of three allows to reduce the integration time of $15 \mathrm{~min}$ to only $5 \mathrm{~min}$, allowing to obtain 3 consecutive data points which push the Nyquist frequency well beyond the highest frequency of the three input signals. However, due to the poor spectral window an unquestionable identification of the 3 input signals will still be difficult. The two right hand side panels repeat the mentioned simulations, but assume that a second satellite will be able to observe the target while it is invisible for the other BRITE. This is the situation we are aiming for with two pairs of BRITE satellites, observing each in the red and blue spectral range. Status as of January 2007. 